\title{
A necessidade de avaliação do impacto econômico da intervenção nutricional na prevenção ou tratamento da doença de Alzheimer: uma revisão narrativa
}

\author{
Shirley Steffany Muñoz Fernández*, Sandra Maria Lima Ribeiro**, Denise Cavallini Cyrillo**
}

\section{Resumo}

As demências afetam mais de 35,6 milhões de pessoas no mundo. Dessas, de $50 \%$ a $70 \%$ são identificadas como prováveis doenças de Alzheimer. A doença de Alzheimer é considerada uma das principais causas de incapacitação, que afeta não só o paciente, mas também seus cuidadores. Além disso, a doença de Alzheimer representa uma alta carga financeira para a sociedade. Atualmente, têm sido propostas alternativas, de natureza farmacológica ou não, que visam postergar o desenvolvimento ou o avanço da doença. Adiar o início da doença, mesmo que por alguns anos, significa a redução desde impacto na economia. Nesse contexto, a prevenção mediante estratégias nutricionais é um importante aspecto a ser investigado. Diversos estudos têm demonstrado que os nutrientes exercem um papel fundamental nas estruturas e no funcionamento do cérebro, são, portanto, importantes nas ações preventivas ou de tratamento da doença de Alzheimer. Com base nessa fundamentação, o objetivo do artigo é realizar uma revisão narrativa, de análise qualitativa, para identificar o impacto econômico das intervenções nutricionais de prevenção ou manejo da doença de Alzheimer. Os achados apontaram falta de evidências da avaliação econômica nesse tipo de intervenções, porém, foi possível extrapolar análises similares realizadas com outras doenças crônicas. Observa-se, em geral, que as estratégias nutricionais, comparadas com outras terapias, são consideradas custo-efetivas.

Palavras-chave: Custo-efetividade. Demência. Doença de Alzheimer. Economia. Nutrição.

* Graduada em Nutrición y Dietética, Universidad del Atlantico, UA, Colômbia. Mestranda do Programa de Pós-Graduação Interunidades em Nutrição Humana Aplicada, Universidade de São Paulo. Endereço para correspondência: Av. Professor Lineu Prestes, 580, Bloco 14, São Paulo, SP, CEP 05508-000. E-mail: shirleymf@usp.br

** Graduada em Nutrição. Doutora em Nutrição Humana Aplicada pela Universidade de São Paulo. Livre docente e professora associada na Escola de Artes Ciências e Humanidades da Universidade de São Paulo. Docente do Programa de Pós-Graduação Interunidades em Nutrição Humana Aplicadada Universidade de São Paulo. E-mail: smlribeiro@usp.br

**** Economista. Doutora em Economia. Professora associada da Faculdade de Economia Administração e Contabilidade. Docente do Programa de Pós-Graduação Interunidades em Nutrição Humana Aplicada. E-mail: dccyrill@usp.br

$\rightarrow$ http://dx.doi.org/10.5335/rbceh.v13i1.5470

Recebido em: 08/10/2015. Aceito em: 24/06/2016. 


\section{Introdução}

As doenças crônicas não transmissíveis (DCNT) constituem a principal causa de mortalidade no mundo (WORLD HEALTH ORGANIZATION, 2011). Essas doenças são especialmente importantes na velhice. Particularmente, as doenças neurodegenerativas são responsáveis pela enorme carga física e emocional projetadas sobre os cuidadores, em geral familiares ou amigos (PRENTICE, 2014). É uma das maiores causas de incapacidade e dependência, características que aumentam à medida que a doença progride (PRINCE; JACKSON, 2009).

A doença de Alzheimer (DA) é caracterizada pela perda irreversível e progressiva da memória e representa a forma mais comum de demência (de $50 \%$ a $75 \%$ dos casos), constituindo a quarta causa de morte em pessoas acima de 65 anos (SONKUSARE; KAUL; RAMARAO, 2005). Conforme estimativas do The World Health Report 2003 (WORLD HEALTH ORGANIZATION, 2003b), a demência contribuiu com $11,2 \%$ de todos os anos vividos com incapacidade nas pessoas com mais de 60 anos, o que representa um índice maior do que $o$ apresentado em outras doenças crônicas degenerativas. A doença é ainda responsável por 17,1 milhões de DALYs (disability-adjusted life years) perdidos (JAMISON et al., 2006). Em 2010, a Federação Internacional das Associações de Alzheimer (Alzheimer Disease International) divulgou dados informando que os custos globais anuais com a demência são significativos, estimando-se para o ano de 2030 um aumento de $85 \%$, em virtude do incremento previsto da incidência dessas doenças (WIMO; PRINCE, 2010).

Estudos recentes indicam que a alimentação, como um fator de risco modificável, pode ter efeitos tanto positivos quanto negativos sobre a saúde ao longo da vida (WORLD HEALTH ORGANIZATION, 2003a), de modo que a intervenção nutricional surge como uma estratégia importante na prevenção e manejo da DA (PRINCE et al., 2014). Em outras palavras, ajustes dos componentes da dieta podem melhorar significativamente a saúde, com consequentes benefícios econômicos. Nesse contexto, a avaliação econômica, ferramenta da Economia da Nutrição que compara os resultados de saúde com os custos das intervenções (como as de caráter nutricional), demonstra ou não essa viabilidade econômica (GYLES et al., 2012).

Em síntese, o aumento na prevalência da DA impacta negativamente tanto a qualidade de vida dos pacientes e de suas famílias como a economia, ao retirar do mercado de trabalho pacientes e seus cuidadores, além de onerar o sistema público de saúde. Sob essa perspectiva, é importante investigar a viabilidade econômica de intervenções nutricionais efetivas no retardo do avanço da DA, com o fim de liberar recursos humanos e o sistema de saúde. Assim, este trabalho investiga as avaliações econômicas (por análise custo-benefício, análise custo-efetividade) das intervenções nutricionais na DA, por meio de uma revisão da literatura. Objetiva-se, assim, conhecer 
com mais profundidade quais são as vantagens e a relação custo-benefício do tratamento nutricional dessa doença (LENOIR-WIJNKOOP et al., 2011), como também seus benefícios na prevenção de outras DCNT (WONG et al., 2011).

\section{Métodos}

A presente revisão foi elaborada a partir do levantamento de artigos nas bases de dados eletrônicas PubMed, Web of Science e Scholar Google, publicados entre os anos 2000 e 2015, em inglês, português e espanhol, empregando os seguintes descritores: doença de Alzheimer, demência, nutrição, nutrientes, economia, análise custo-efetividade, doença crônica. Como critérios de inclusão foram selecionadas pesquisas sobre avaliação econômica de intervenção nutricional para a prevenção ou o tratamento com nutrientes ou dietas na DA ou demência; foram excluídos estudos de avaliação econômica na DA de intervenções não nutricionais, por exemplo, medicamentosas ou com terapias alternativas. Os resultados foram analisados de forma qualitativa mediante descrição narrativa.

\section{Epidemiologia}

As estatísticas sugerem que a incidência das demências nos idosos aumentará progressivamente, sobretudo nos países de maior crescimento demográfico. O Delphi Consensus Study estimou, em 2001, uma prevalência global de demência de 24,3 milhões de pessoas, em catorze regiões do mundo, com uma incidência anual de 4,6 milhões
(FERRI et al., 2005). Posteriormente, em 2010, realizou-se nova estimativa dessa prevalência, com o estudo Global Burden of Disease, em 21 regiões de todo o mundo, que determinou um total de 35,6 milhões de casos; com a previsão de que esses números dobrem a cada vinte anos (PRINCE et al., 2013).

De acordo com as estimativas de mortalidade da Organização Mundial da Saúde, a DA era a vigésima colocada entre as principais causas de morte em nível global no ano de 2012 (WORLD HEALTH ORGANIZATION, 2014) e a quarta causa de morte nos países de renda alta, com uma taxa de mortalidade de 42 por 100.000 habitantes (WORLD HEALTH ORGANIZATION, 2012). Na região das Américas, a doença teve um progresso expressivo, ao passar da décima causa de morte no ano 2000 , com uma taxa de mortalidade de 12,7 mortes por 100.000 habitantes $(1,9 \%)$, para a terceira principal causa em 2012 , com taxa de 33,3 mortes por 100.000 habitantes $(4,9 \%$ ) (WORLD HEALTH ORGANIZATION, 2014). O Brasil está entre os nove países de maior prevalência, com cerca de um milhão de casos (PRINCE et al., 2013).

\section{0 impacto econômico da doença de Alzheimer}

A demência representa uma alta carga econômica para a sociedade, que incrementa à medida que há a tendência de aumento da população idosa. Além dos custos associados diretamente ao tratamento (consultas médicas, medica- 
mentos e internações), a demência exige cuidados que vão desde a ajuda para as atividades instrumentais de vida diária (como cozinhar ou fazer compras) até o cuidado pessoal completo, que pode ser prestado por familiares, amigos, por serviços assistenciais ou de home care. Além disso, a demência impõe custos adicionais à sociedade, uma vez que retira da vida ativa indivíduos que ainda podem contribuir para a produção: pacientes e cuidadores. Nesse contexto, o custo social da demência abrange gastos explícitos (custos diretos de tratamento médico e de assistência social) e gastos implícitos (a perda de produtividade do indivíduo que adquire a doença e, também, a de seus cuidadores). A maioria dos trabalhos de avaliação do ônus econômico da demência é produzido nos países desenvolvidos; países em desenvolvimento ainda têm poucas avaliações do assunto (WORLD HEALTH ORGANIZATION, 2006).

Em 2010, a Alzheimer's Disease International promoveu um estudo sobre o impacto econômico global da demência que estimou um custo total em nível mundial de US $\$ 604$ bilhões (Tabela 1). Esses custos representavam cerca de $1 \%$ do produto interno bruto do mundo, com variações de $0,24 \%$ em países de baixa renda, $0,35 \%$ em países de renda média baixa, $0,50 \%$ em países de renda média alta e $1,24 \%$ em países de alta renda. Os países de baixa renda, onde residem $14 \%$ das pessoas com a doença, foram responsáveis por menos de $1 \%$ dos custos totais. Países de renda média, com $40 \%$ da prevalência, contribuíram com $10 \%$ dos custos. E países de renda alta, com $89 \%$ dos custos e $46 \%$ da prevalência. De acordo com o relatório o custo per capita, associado à demência foi de US\$ 868 nos países de baixa renda, US\$ 3.109 nos de renda média baixa, US\$ 6.827 nos de renda média alta e US $\$ 32.865$ em países de alta renda. Essa discrepância está associada à composição dos custos. Ressalta-se a constatação de que os custos informais e de assistência social representam a maior proporção do custo total, $41,7 \%$ e $42,3 \%$, respectivamente, enquanto que os custos diretos médicos representam 16,0\%. Essa proporção era maior entre os países de baixa e média renda do que nos de alta renda (WIMO; PRINCE, 2010).

Tabela 1 - Custos agregados do impacto econômico global da demência segundo regiões no mundo

\begin{tabular}{l|r}
\hline \multicolumn{1}{c|}{ Região } & \multicolumn{1}{c}{$\begin{array}{c}\text { Custo total } \\
\text { (bilhões US } \$ \text { ) }\end{array}$} \\
\hline Austrália e Oceania & 10,18 \\
Ásia & 113,49 \\
Europa & 238,64 \\
América do Norte & 213,04 \\
América Latina e Caribe & 22,80 \\
África & 5,84 \\
Total & 603,99 \\
\hline
\end{tabular}

Fonte: adaptado de WIMO; PRINCE, 2010.

Pesquisas já demonstraram que retardar o início ou a progressão da demência pode resultar em uma diminuição significativa dos custos da doença (BRODATY et al., 2011). Por exemplo, 
postergar por um mês a institucionalização de um paciente com DA moderada a grave resultaria em uma redução mensal no orçamento do sistema de saúde de US\$ 1.863 (ZHU; SANO, 2006). Os tratamentos farmacológicos também envolvem gastos consideráveis em longo prazo, devido ao caráter crônico da doença. Além disso, os efeitos adversos dos medicamentos sobre a progressão da doença ainda não foram determinados, o que suscita controvérsias a respeito do seu custo-efetividade na justificativa desse ônus econômico (CASEY; ANTIMISIARIS; O'BRIEN, 2010). Por conseguinte, cabe investigar a efetividade de intervenções preventivas alternativas que retardem o surgimento e a progressão da doença.

\section{A nutrição como estratégia preventiva da DA}

As transições demográfica e nutricional que ocorreram ao longo dos anos, somadas ao uso de tabaco e à inatividade física, acarretaram o aumento da prevalência de DCNT (BELAHSEN, 2014). A partir disso, tem-se constatado que a nutrição, por ser um dos principais fatores modificáveis, constitui-se estratégia essencial na prevenção e tratamento dessas doenças (PUSKA, 2002). Ademais, há evidências de que existe uma relação biológica entre a má nutrição no início da vida e DCNT na idade adulta (HOFFMAN, 2010).

Atualmente, não existe cura para a demência nem um tratamento que modifique o seu curso. Entretanto, pesquisas epidemiológicas sugerem vários fatores de risco e de proteção para a DA, abrindo perspectivas para a prevenção ou a redução de seu risco (WIMO et al., 2014). No cenário clínico, a prevenção primária e as intervenções terapêuticas para a DA concentram-se, principalmente, em modificações de estilo de vida, principalmente a partir da quarta década de vida (OBOUDIYAT et al., 2013). Há evidências de que a intervenção nutricional antes do início da demência pode levar a melhorias na saúde geral (RAMESH et al., 2010).

$\mathrm{O}$ estado nutricional e a função cognitiva estão correlacionados e servem de base fundamental para determinar 0 estado funcional e de saúde em idosos. Tem-se demonstrado que o aumento do risco de má nutrição, principalmente de deficiências subclínicas (por exemplo, folato, vitamina B12), pode prejudicar a cognição, o humor e, em consequência, o apetite. Ademais, variações na ingestão de alimentos podem ser refletidas em mudanças nos tecidos do cérebro e no desempenho cognitivo (NYARADI et al., 2013). Pesquisas indicam que o estresse oxidativo e o acúmulo de radicais livres derivados da peroxidação lipídica estão envolvidos na fisiopatologia da doença e podem acelerar a degeneração de neurônios (CHEN; ZHONG, 2014; SULTANA; PERLUIGI; ALLAN BUTTERFIELD, 2013; WANG et al., 2014). De outro modo, alguns nutrientes (vitaminas e ácidos graxos) têm efeito antioxidante e contribuem na diminuição do estresse oxidativo no cérebro. Ainda, alguns nutrientes modulam a produção e a atividade de neurotrofinas (proteínas 
implicadas no desenvolvimento, função e sobrevivência dos neurônios), com efeitos vasoprotetores, o que favorece a depuração da proteína $\beta$-amiloide, cujo acúmulo no cérebro tem sido considerado como característica inicial da doença. Estudos são desenvolvidos com diferentes nutrientes e padrões alimentares para confirmar a relação entre micro e macronutrientes e a função cognitiva. Contudo, ainda não foram obtidas evidências suficientes (PRINCE et al., 2014).

Ainda, no que diz respeito ao manejo de pacientes que já iniciaram o desenvolvimento da $\mathrm{DA}$, os cuidados alimentares, o apoio da família e um estilo de vida mais saudável são, provavelmente, as melhores estratégias para melhorar a qualidade de vida, tanto dos doentes quanto de cuidadores (BARNARD et al., 2014).

\section{Aspectos econômicos dos benefícios nutricionais}

A avaliação econômica de intervenções nutricionais relaciona o resultado da intervenção e seus custos, com o objetivo de determinar, em média, o investimento necessário para alcançar uma unidade de resultado. Essa análise é feita por meio de diversos métodos, como análise custo-benefício ou custo-efetividade, que permitem, em determinadas circunstâncias, comparações de investimentos em nutrição com aqueles feitos em outros aspectos dos cuidados em saúde (WONG et al., 2011). Esses métodos têm sido pouco aplicados nos trabalhos de pesquisa de intervenção nutricional.

A intervenção nutricional, vista como estratégia primária de prevenção de DCNT, possibilita a melhora da saúde (KOPONEN et al., 2012), além de ter a vantagem de ser custo-efetiva de fácil implementação e aceitação pela sociedade, com baixo risco de eventos adversos (SWAMINATHAN; JICHA, 2014). Essa estratégia poderia ser desenhada com base na análise e na caracterização dos resultados em saúde e, também, os econômicos, no que diz respeito a intervenções nutricionais para prevenção da doença.

Com relação à DA, na pesquisa bibliográfica realizada para este estudo, foram localizadas avaliações econômicas de tratamentos farmacológicos, métodos diagnóstico e intervenções não farmacológicas como terapia de estimulação cognitiva e exercício físico (KNAPP; IEMMI; ROMEO, 2013; POURYAMOUT et al., 2012; WIMO et al., 2014). Entretanto, não foram encontrados estudos que determinassem a razão custo efetividade de intervenções nutricionais com vistas a prevenir ou a retardar o início da DA, embora tenham sido descobertas pesquisas de análise econômica nutricional em outras doenças crônicas não transmissíveis, o que, de certa forma, respalda a viabilidade dessa avaliação econômica na DA. Na Tabela 2 , são descritos os principais estudos encontrados nesse contexto. 
Tabela 2 - Principais estudos de análise econômico-nutricional em doenças crônicas não transmissíveis

\begin{tabular}{|c|c|c|c|c|c|}
\hline Referência & $\begin{array}{l}\text { Desenho do } \\
\text { estudo }\end{array}$ & $\begin{array}{l}\text { Situação } \\
\text { clínica }\end{array}$ & Tipo de intervenção & $\begin{array}{l}\text { Tipo de } \\
\text { análise }\end{array}$ & Principais resultados \\
\hline $\begin{array}{l}\text { Mennini } \\
\text { et al., } 2014\end{array}$ & $\begin{array}{l}\text { Modelo de } \\
\text { Markov }^{1}\end{array}$ & $\begin{array}{l}\text { Doença } \\
\text { renal } \\
\text { crônica }\end{array}$ & $\begin{array}{l}\text { Dieta muito baixa em } \\
\text { proteína comparada } \\
\text { com uma dieta mode- } \\
\text { radamente baixa em } \\
\text { proteínas }\end{array}$ & $\begin{array}{l}\text { ACE, } \\
\text { QALY }\end{array}$ & $\begin{array}{l}\text { O tratamento dietético melho- } \\
\text { rou } 0,09 \text { QALY após dois anos, } \\
0,16 \text { após três anos, } 0,36 \text { após } \\
\text { cinco anos e até } 0,93 \text { QALY in- } \\
\text { crementais após os dez anos. } \\
\text { Após dois anos, o modelo es- } \\
\text { timado de custo incrementou } \\
\text { em favor do tratamento dieté- } \\
\text { tico mais restritivo de } € 826 \text {, } \\
€ 2.146 \text {, } € 5.203 \text { e } € 10.375 \\
\text { para dois, três, cinco e dez } \\
\text { anos de seguimento, respec- } \\
\text { tivamente. }\end{array}$ \\
\hline $\begin{array}{l}\text { Panagiotakos } \\
\text { et al., } 2007\end{array}$ & $\begin{array}{l}\text { Estudo } \\
\text { transversal } \\
\text { com análise } \\
\text { econômica }\end{array}$ & $\begin{array}{l}\text { Doença } \\
\text { cardíaca } \\
\text { coronária }\end{array}$ & Adesão à $\mathrm{MeDi}^{2}$ & Racei & $\begin{array}{l}\text { O risco coronariano em dez } \\
\text { anos maior de } 10 \% \text { foi de } \\
4,2 \% \text { em participantes pró- } \\
\text { ximos à MeDi, e de } 39,8 \% \\
\text { naqueles distantes desse } \\
\text { padrão ( } P<0,001) \text {. O custo } \\
\text { total de saúde foi } € 336,720 \\
\text { nos distantes e } € 35.880 \text { nos } \\
\text { mais próximos. Os anos de } \\
\text { vida perdidos por deficiência } \\
\text { foi } 6,8 \text { nos distantes e } 0,9 \\
\text { nos próximos. A Racei foi de } \\
€ 50.989 \text {. }\end{array}$ \\
\hline $\begin{array}{l}\text { Rajgopal } \\
\text { et al., } 2002\end{array}$ & $\begin{array}{l}\text { Estudo } \\
\text { retrospectivo de } \\
\text { custo-benefício }\end{array}$ & $\begin{array}{l}\text { Doenças } \\
\text { crônicas }\end{array}$ & $\begin{array}{l}\text { Programa de educa- } \\
\text { ção nutricional }\end{array}$ & $\mathrm{ACB}^{3}$ & $\begin{array}{l}\text { Razão inicial custo-benefício } \\
\text { U\$ } \$ 10,64 / \cup \$ \$ 1,00 ; \text { com ra- } \\
\text { zões de análise de sensibili- } \\
\text { dade subsequente produtoras } \\
\text { que variam de U\$\$2,66/1,00 } \\
\text { a U\$ } \$ 17,04 / 1,00 \text {. }\end{array}$ \\
\hline Tice et al, 2001 & $\begin{array}{l}\mathrm{ACE}^{4} \text { usando } \\
\text { o modelo } \\
\text { da Política } \\
\text { de Doença } \\
\text { Coronária }\end{array}$ & $\begin{array}{l}\text { Eventos } \\
\text { coronários }\end{array}$ & $\begin{array}{l}\text { Dieta com cereais enri- } \\
\text { quecidos para aumen- } \\
\text { to de B9 por } 100 \mathrm{~g} / \mathrm{d} \text {, } \\
\text { comparada com a mes- } \\
\text { ma dieta sem adição } \\
\text { de B9; e terapia com } 1 \\
\text { mg de B9 e } 0,5 \mathrm{mg} \text { de } \\
\text { B12 comparada a dieta } \\
\text { com grãos fortificados } \\
\text { com B9. }\end{array}$ & ACE & $\begin{array}{l}\text { A fortificação de grão com } \\
\text { vitaminas B9 e B12 para tra- } \\
\text { tamento de pacientes com } \\
\text { DC conhecida durante dez } \\
\text { anos resultaria em } 310.000 \\
\text { menos mortes e custos mais } \\
\text { baixos. Em sujeitos sem DC } \\
\text { conhecida, a suplementação } \\
\text { de ácido fólico e cianocobala- } \\
\text { mina adicional à fortificação, } \\
\text { foram projetadas salvar mais } \\
\text { de } 300.000 \text { QALYs, e mais de } \\
\text { US\$ } 2 \text { bilhões, em homens } \\
\text { acima de } 45 \text { anos, e mais de } \\
140000 \text { QALYs em mulheres } \\
\text { acima de } 55 \text { anos, ao longo de } \\
\text { dez anos. }\end{array}$ \\
\hline
\end{tabular}

Fonte: elaborada pelos autores com base em dados da pesquisa.

Nota: 1 - Análise de simulação probabilística de possíveis eventos; 2 - MeDi, dieta mediterrânea; 3 - ACB, Análise de custo-benefício; 4 - ACE, Análise de custo-efetividade; ACU, Análise de custo-utilidade; QALY, quality-adjusted life-iear (anos de vida ajustados por qualidade); Racei, Razão de análise de custo-efetividade incremental; B9, ácido fólico; B12, cianocobalamina; DC, doenças coronárias. 
Dentre as possíveis alternativas de abordagem nutricional, o padrão mediterrâneo de dieta tem sido amplamente investigado nos pacientes com DA e com risco de demência, mostrando que a aderência a esse padrão alimentar pode reduzir o risco de desenvolvimento e da evolução da DA (THAIPISUTTIKUL; GALVIN, 2012). Tal resultado, porém, não foi associado aos custos da intervenção. Há evidências que sustentam as vantagens econômicas e em saúde da intervenção nutricional em outras doenças crônicas (DALZIEL; SEGAL, 2007). Por exemplo, uma revisão sistemática comparou o custo e o custo-efetividade da adesão à dieta mediterrânea com uma dieta ocidental em pacientes após o primeiro infarto agudo do miocárdio (IAM). Os resultados demonstraram que, ao longo de dez anos, a dieta mediterrânea resultou em um custo incremental por QALY (quality-adjusted life-year, anos de vida ajustados por qualidade) ganho de US\$ 703 por pessoa, com uma razão de custo-efetividade de US\$2.500 por IAM não fatal evitado e US $\$ 4.000$ por morte evitada. A intervenção por meio da dieta mediterrânea, somada a outras mudanças no estilo de vida com o fim de prevenir diabetes teve um ótimo desempenho: US\$ 760 e US $\$ 1.410$ por QALY ganho, respectivamente (SAULLE; SEMYONOV; LA TORRE, 2013). Outro estudo de adesão à dieta mediterrânea na doença coronária (DC) evidenciou o risco coronariano de aproximadamente $10 \%$ em dez anos em 4,2\% dos participantes com dietas mais próximas à mediterrânea (acima do escore mediano da dieta); naqueles com alimentação mais distante desse padrão, o risco estimado foi de 39,8\% $(\mathrm{P}<0,001)$. Ademais, os participantes com o padrão alimentar mais próximo da mediterrânea tinham $43 \%$ (OR 0,57, 95\% IC 0,38-0,86) menor probabilidade de sofrer um evento coronário em dez anos superior a 10\%. Esse estudo estimou ainda o custo total dos cuidados de saúde em $€ 336.720$ para aqueles que estavam distantes do padrão mediterrâneo, e $€ 35.880$ para aqueles que estavam mais próximos. Os anos de vida perdidos por incapacidade foram, em média, 6,8 para o grupo que não adotou a dieta mediterrânea, ao passo que para aqueles que apresentavam uma alimentação mais próxima à da mediterrânea a média de anos de vida perdidos foi de apenas 0,9 (PANAGIOTAKOS et al., 2007).

Uma pesquisa do Serviço Nacional de Saúde italiano, que usou um modelo de simulação probabilística em pacientes com doença renal crônica indicou que o tratamento com uma dieta com muito baixo conteúdo proteico é eficaz em relação aos custos daquelas moderadamente baixas em proteínas. $\mathrm{O}$ custo anual de diálise por paciente foi de cerca de $€ 34.072$ e o custo de uma dieta muito baixa em proteína foi de $€ 1.440$ por paciente por ano. O tratamento dietético melhorou 0,09 QALY após dois anos, 0,36 após cinco anos e até 0,93 QALY incrementais após os primeiros dez anos. Depois de dois anos, o modelo estimou um custo incremental em favor do tratamento dietético mais restritivo de $€ 826$, $€ 2.146$, $€ 5.203$ e $€ 10.375$ para dois, três, cinco e 
dez anos de seguimento, respectivamente (MENNINI et al., 2014).

Outro estudo, usando o modelo da Política de Doença Cardíaca Coronária, dos Estados Unidos, demonstra que a suplementação com ácido fólico e cianocobalamina, além de ter efeitos benéficos na redução dos níveis de homocisteína associados à patogênese das doenças vasculares, contribui na prevenção dessas doenças e pode ser custo-efetiva entre algumas populações. A fortificação de cereais com ácido fólico foi prevista para a diminuição de eventos coronarianos em $8 \%$ para mulheres e $13 \%$ para homens. O tratamento com ácido fólico e cianocobalamina, restrita à fortificação de grãos, em pacientes com DC durante período de dez anos poderia reduzir em 310.000 o número de óbitos e os custos associados. Para a suplementação, além da fortificação de grãos, durante o mesmo tempo, em homens acima de 45 anos e mulheres acima de 55, sem DC conhecida, a projeção foi de poupar mais de 300.000 QALYs e mais de US\$ 2 bilhões no caso dos homens, e 140.000 QALYs nas mulheres (TICE et al., 2001).

Um estudo de análise custo-benefício de um Programa de Educação Nutricional e Alimentar para a prevenção de doenças crônicas obteve como resultado uma razão custo-benefício inicial de US $\$ 10.64 / \$ 1.00$ (a análise de sensibilidade produziu razões de US\$2.66/ US $\$ 1.00$ a US $\$ 17.04 / U S \$ 1.00)$. A razão inicial indica que para cada dólar gasto no programa, existe a possibilidade de que mais de 10 dólares possam ser poupados no custo futuro em cuidados com a saúde. Um aspecto desse estudo que merece atenção é o fato de viabilizar a valoração dos benefícios de um programa de educação nutricional (RAJGOPAL et al., 2002).

Essas evidências destacam os resultados favoráveis da intervenção nutricional para a saúde na prevenção de doenças crônicas, melhoria na qualidade de vida e aumento da expectativa de vida, na medida em que repercute em ganho líquido para o sistema de saúde com redução dos custos totais. Ainda assim, a falta de resultados específicos da avaliação econômica de terapias nutricionais sobre a demência limita as resposta às questões inicialmente apontadas nesta revisão, que têm o objetivo de determinar a relação de custo-efetividade da intervenção nutricional nessa doença e os benefícios ao sistema de saúde e à população em geral. Outro aspecto que provavelmente limita a estimativa desta análise econômica pode ser atribuído ao fato de que ainda não foi definido um tratamento nutricional para as demências. Além disso, pode-se citar a falta de políticas públicas consistentes, em nível nacional e internacional, que incentivem e suportem na prática clínica essas estratégias para a prevenção de demências (BARBOSA ABREU; VAL, 2015; WORLD HEALTH ORGANIZATION, 2012). 


\section{Conclusões}

A incidência de DA aumenta progressivamente com o envelhecimento populacional. Portanto, há um incremento dos custos associados aos cuidados de pacientes. Dessa forma é necessário elaborar estudos de análise econômica das intervenções nessa doença, com o fim de avaliar a razão custo-efetividade ou a razão custo-benefício dos tratamentos que visam retardar o início da DA.

Este trabalho destaca a relevância da Economia da Nutrição, na medida em que abrange as técnicas para a realização de avaliações econômicas de intervenções que atenuam a sintomatologia e a progressão da DA e evidenciam a importância da nutrição como estratégia fundamental para a prevenção, o tratamento e a redução da carga econômica da doença. Novos estudos são necessários, no sentido de avaliar os aspectos econômicos das intervenções nutricionais especificamente na DA, e que forneçam evidências mais robustas, com dados específicos, que determinem os efeitos para a saúde e para economia, em termos de benefícios e de riscos potenciais.

Atualmente, não há dados de custo-efetividade das estratégias nutricionais na DA em comparação a terapias tradicionais com medicamentos ou a nenhuma intervenção. Todavia, foram localizados estudos capazes de avaliar o impacto econômico da dieta em relação a outras doenças crônicas, o que permitiu concluir que intervenções nutricionais podem vir a ser custo-efetivas no tratamento da DA. É esperado que a prevenção ou a inter- venção nutricional traga benefícios tanto no bem-estar e na saúde dos pacientes e seus cuidadores, quanto na redução dos ônus totais em longo prazo.

A realização de futuras pesquisas que abordem esses temas são cruciais para a tomada de decisões na área clínica, saúde pública e políticas econômicas. Atingir essa meta pode exigir reorganização dos modelos de gasto com saúde para gerar evidências relevantes na implementação de iniciativas e uma sólida implantação dos hábitos alimentares nas estruturas sociais.

\section{The need of assessment of economic burden of nutrition intervention in the prevention or treatment of Alzheimer's disease: a narrative review}

\section{Abstract}

Dementia affects more than 35,6 million people worldwide, which 50 to $70 \%$ are diagnosed as probable Alzheimer's disease (AD). AD is considered one of leading causes of disability in the elderly, affecting not only the patient, but also caregivers. Furthermore, this condition represents a high economic burden for the whole society. Currently, drug or non-drug strategies have been proposed, to delay the early onset of the disease. Slowing down the beginning of symptoms of the disease, even for a few years, certainly will mean reduction of the economic impact caused. In this context, prevention of the onset or progress of $\mathrm{AD}$ through nutritional strategies may be a significant issue to be investigated. Several researches have shown that nutrients perform an important role in the brain structures and function; therefore, it may be important in the prevention or 
management of the AD. In accordance with this, the aim of this review is to identify the economic impact of nutrition interventions in prevention or treatment of $\mathrm{AD}$, through a qualitative analysis in a narrative review. Findings show the lack of evidences in economical assessment of this intervention in AD. Nonetheless, notably most nutrition strategies are cost-effective, compared to other pharmacological and non-pharmacological therapies, in the treatment of chronic diseases, which eventually might be extrapolated in the nutritional treatment of $\mathrm{AD}$.

Keywords: Cost-effectiveness. Dementia. Alzheimer's disease. Nutrition. Economy.

\section{Agradecimentos}

À Coordenação de Aperfeiçoamento de Pessoal de Nível Superior pelo apoio financeiro.

\section{Referências}

BARBOSA ABREU, C.; VAL, E. M. Políticas públicas de saúde para idosos com Alzheimer. Novos Estudos Jurídicos, Itajaí, v. 20, n. 2, p. 727-754, maio/ago. 2015.

BARNARD, N. D. et al. Dietary and lifestyle guidelines for the prevention of Alzheimer's disease. Neurobiology of Aging, v. 35, p. S74S78, May 2014.

BELAHSEN, R. Nutrition transition and food sustainability. Proceedings of the Nutrition Society, v. 73, n. 3, p. 385-388, May 2014.

BRODATY, H. et al. The world of dementia beyond 2020. Journal of the American Geriatrics Society, v. 59, n. 5, p. 923-927, May 2011.

CASEY, D. A.; ANTIMISIARIS, D.; O'BRIEN, J. Drugs for Alzheimer's disease: are they effective? $P \&$ T: a peer-reviewed journal for formulary management, v. 35, n. 4, p. 208-211, Apr. 2010.
CHEN, Z.; ZHONG, C. Oxidative stress in Alzheimer's disease. Neuroscience Bulletin, v. 30, n. 2, p. 271-281, Apr. 2014.

DALZIEL, K.; SEGAL, L. Time to give nutrition interventions a higher profile: costeffectiveness of 10 nutrition interventions. Health Promotion International, v. 22, n. 4, p. 271-283, $1^{\text {th }}$ Dec. 2007.

FERRI, C. P. et al. Global prevalence of dementia: s Delphi consensus study. Lancet, v. 366, n. 9.503, p. 2.112-2.117, Dec. 2005.

GYLES, C. L. et al. Health economics and nutrition: a review of published evidence. Nutrition Reviews, v. 70, n. 12, p. 693-708, Dec. 2012.

HOFFMAN, D. J. Early nutrition and adult health: perspectives for international and community nutrition programs and policies. Nutrition Research and Practice, v. 4, n. 6, p. 449-454, Dec. 2010.

JAMISON, D. T. et al. (Ed). Cost-effective strategies for noncommunicable diseases, risk factors, and behaviors. In:

Priorities in Health. Washington (DC): World Bank, 2006. p. 97-128. Disponível em: <http://www.ncbi.nlm.nih.gov/books/ NBK10246/>. Acesso em: 1ํ dez. 2014.

KNAPP, M.; IEMMI, V.; ROMEO, R. Dementia care costs and outcomes: a systematic review. International Journal of Geriatric Psychiatry, v. 28, n. 6, p. 551-561, Jun. 2013.

KOPONEN, A. et al. Nutrition economics: towards comprehensive understanding of the benefits of nutrition. Microbial Ecology in Health and Disease, v. 23, n. 18.585, p. 46-50, Jan. 2012.

LENOIR-WIJNKOOP, I. et al. Nutrition economics - characterising the economic and health impact of nutrition. The British Journal of Nutrition, v. 105, n. 1, p. 157-166, Jan. 2011.

MENNINI, F. S. et al. Economic effects of treatment of chronic kidney disease with low-protein diet. Journal of Renal Nutrition, v. 24, n. 5, p. 313-321, Sept. 2014. 
NYARADI, A. et al. The role of nutrition in children's neurocognitive development, from pregnancy through childhood. Frontiers in Human Neuroscience, v. 7, n. 97, p. 1-16, Mar. 2013.

OBOUDIYAT, C. et al. Alzheimer's disease. Seminars in neurology, v. 33, n. 4, p. 313-329, Sept. 2013.

PANAGIOTAKOS, D. et al. Estimating the 10-year risk of cardiovascular disease and its economic consequences, by the level of adherence to the Mediterranean diet: the ATTICA study. Journal of Medicinal Food, v. 10, n. 2, p. 239-243, Jun. 2007.

POURYAMOUT, L. et al. Economic evaluation of treatment options in patients with Alzheimer's disease: a systematic review of cost-effectiveness analyses. Drugs, v. 72, n. 6, p. 789-802, 16 Apr. 2012.

PRENTICE, A. M. Nutrition and chronic disease: lessons from the developing and developed world. In: BLACK, R. E.; SINGHAL, A.; UAUY, R. (Ed). International nutrition: achieving millennium goals and beyond. Nestlé Nutrition Institute workshop series, Basel, v. 78, p. 155-160, 27 Jan. 2014. Disponível em: <http://www.ncbi.nlm.nih.gov/ pubmed/24504216>. Acesso em: 10 out. 2014 .

PRINCE, M. et al. The global prevalence of dementia: a systematic review and metaanalysis. Alzheimer's \& dementia: The Journal of the Alzheimer's Association, v. 9, n. 1, p. 63-75.e2, Jan. 2013.

Nutrition and dementia: a review of available research. London: Alzheimer's Disease International, Feb. 2014. p. 1-84. Disponível em: <https://www.alz.co.uk/sites/ default/files/pdfs/nutrition-and-dementia. pdf>. Acesso em: 4 dez. 2014.

PRINCE, M.; JACKSON, J. (Ed). World Alzheimer report 2009. London: Alzheimer's Disease International, 2009. 96 p. Disponível em: <http://www.alz.co.uk/research/files/ WorldAlzheimerReport.pdf>. Acesso em: 22 set. 2014.
PUSKA, P. Nutrition and global prevention on non-communicable diseases. Asia Pacific Journal of Clinical Nutrition, v. 11, n. s9, p. S755-S758, Dec. 2002.

RAJGOPAL, R. et al. Cost-benefit analysis indicates the positive economic benefits of the Expanded Food and Nutrition Education Program related to chronic disease prevention. Journal of nutrition education and behavior, v. 34, n. 1, p. 26-37, Jan./Feb. 2002.

RAMESH, B. N. et al. Neuronutrition and Alzheimer's disease. Journal of Alzheimer's Disease, v. 19, n. 4, p. 1.123-1.139, Jan. 2010.

SAULLE, R.; SEMYONOV, L.; LA TORRE, G. Cost and cost-effectiveness of the mediterranean diet: Results of a systematic review. Nutrients, v. 5, n. 11 , p. 4.566-4.586, Nov. 2013.

SONKUSARE, S. K.; KAUL, C. L.; RAMARAO, P. Dementia of Alzheimer's disease and other neurodegenerative disordersmemantine, a new hope. Pharmacological Research, v. 51, n. 1, p. 1-17, Jan. 2005.

SULTANA, R.; PERLUIGI, M.; ALLAN BUTTERFIELD, D. Lipid peroxidation triggers neurodegeneration: A redox proteomics view into the Alzheimer disease brain. Free Radical Biology and Medicine, v. 62, p. 157-169, Sept. 2013.

SWAMINATHAN, A.; JICHA, G. A. Nutrition and prevention of Alzheimer's dementia. Frontiers in Aging Neuroscience, v. 6, n. 282, p. 1-13, 20 Jan. 2014.

THAIPISUTTIKUL, P.; GALVIN, J. E. Use of medical foods and nutritional approaches in the treatment of Alzheimer's disease. Clinical practice, London, v. 9, n. 2, p. 199-209, Mar. 2012.

TICE, J. A. et al. Cost-effectiveness of vitamin therapy to lower plasma homocysteine levels for the prevention of coronary heart disease: effect of grain fortification and beyond. JAMA, v. 286, n. 8, p. 936-943, Jan. 2001. 
WANG, X. et al. Oxidative stress and mitochondrial dysfunction in Alzheimer's disease. Biochimica et Biophysica Acta, v. 1.842, n. 8, p. 1.240-1.247, 2014.

WIMO, A. et al. Health economic evaluation of treatments for Alzheimer's disease: impact of new diagnostic criteria. Journal of Internal Medicine, v. 275, n. 3, p. 304-316, 8 Mar. 2014.

WIMO, A.; PRINCE, M. World Alzheimer report 2010: the global economic impact of dementia. London: Alzheimer's Disease International, Sept. 2010. p. 23-26. Disponível em: <http://www.alz.org/documents/national/ world_alzheimer_report_2010.pdf>. Acesso em: 23 set. 2014 .

WONG, J. B. et al. Economic analysis of nutrition interventions for chronic disease prevention: methods, research, and policy. Nutrition Reviews, v. 69, n. 9, p. 533-549, 2011.

WORLD HEALTH ORGANIZATION. Diet, nutrition and the prevention of chronic diseases: report of the joint WHO/FAO expert consultation. Geneva: WHO Press, 2003a. (WHO Technical Report Series, n. 916). p. 1-149. Disponível em: <http://www.who. int/dietphysicalactivity/publications/trs916/ download/en/>. Acesso em: 10 out. 2014.

. Global status report on noncommunicable diseases 2010. Geneva: WHO Press, 2011. p. 1-162. WHO Library Cataloguingin-Publication Data. Disponível em: <http:// www.who.int/nmh/publications/ncd_report_ full_en.pdf>. Acesso em: 22 set. 2014.

. Global Health Estimates 2014 Summary Tables: Deaths by Cause, Age and Sex, By Who Region, 2000-2012. Geneva, Switzerland: WHO, 2014. Disponível em: <http:/www.who.int/healthinfo/global_burden_disease/en/>. Acesso em: 24 nov. 2014.

. Neurological disorders: public health challenges. Geneva: WHO Press, 2006. p. 1-128. WHO Library Cataloguingin-Publication Data. Disponível em: <http:// www.who.int/mental_health/neurology/neurological_disorders_report_web.pdf $>$. Acesso em: 25 set. 2014.

The world health report 2003: Shaping the future. Geneva: WHO, 2003b. Disponível em: <http://www.who.int/ whr/2000/en/whr00_en.pdf>. Acesso em: $1^{\circ}$ dez. 2014

Dementia: a public health priority. Geneva: WHO Press, 2012. p. 1-102. WHO Library Cataloguing-in-Publication Data. Disponível em: <http:/whqlibdoc.who.int/ publications/2012/9789241564458_eng.pdf>. Acesso em: 23 set. 2014.

ZHU, C. W.; SANO, M. Economic considerations in the management of Alzheimer. Clinical Interventions in Aging, v. 1, n. 2, p. 143-154, 2006. 